\title{
Fbxw7 acts as a critical fail-safe against premature loss of hematopoietic stem cells and development of T-ALL
}

\author{
Sahoko Matsuoka, ${ }^{1,2}$ Yuichi Oike, 1,3,4,13 \\ Ichiro Onoyama, ${ }^{5}$ Atsushi Iwama, ${ }^{6,7}$ Fumio Arai, ${ }^{1}$ \\ Keiyo Takubo, ${ }^{1}$ Yoichi Mashimo, ${ }^{8}$ \\ Hideyuki Oguro, ${ }^{7}$ Eriko Nitta, ${ }^{1}$ Keisuke Ito, ${ }^{1,2}$ \\ Kana Miyamoto, ${ }^{1}$ Hiroki Yoshiwara, ${ }^{1}$ \\ Kentaro Hosokawa, ${ }^{1}$ Yuka Nakamura, ${ }^{1}$ \\ Yumiko Gomei, ${ }^{1}$ Hiroko Iwasaki, ${ }^{1}$ \\ Yasuhide Hayashi,' Yumi Matsuzaki, ${ }^{10}$ \\ Keiko Nakayama, ${ }^{11}$ Yasuo Ikeda, $^{2}$ Akira Hata, ${ }^{7}$ \\ Shigeru Chiba, ${ }^{12}$ Keiichi I. Nakayama, ${ }^{5}$ and \\ Toshio Suda ${ }^{1,6,14}$ \\ ${ }^{1}$ Department of Cell Differentiation, The Sakaguchi \\ Laboratory, School of Medicine, Keio University, Tokyo \\ 160-8582, Japan; ${ }^{2}$ Department of Internal Medicine, School of \\ Medicine, Keio University, Tokyo 160-8582, Japan; ${ }^{3}$ PRESTO, \\ Japan Science Technology Agency (JST), Saitama 322-0012, \\ Japan; ${ }^{4}$ Department of Molecular Genetics, Graduate School \\ of Medical Sciences, Kumamoto University, Kumamoto \\ 860-8556, Japan; ${ }^{5}$ Department of Molecular and Cellular \\ Biology, Medical Institute of Bioregulation, Kyushu \\ University, Fukuoka 812-8582, Japan; ${ }^{6}$ CREST, Japan Science \\ Technology Agency (JST), Saitama 322-0012, Japan; \\ ${ }^{7}$ Department of Cellular and Molecular Medicine, Chiba \\ University, Chiba 260-8670, Japan; ${ }^{8}$ Department of Public \\ Health, Graduate School of Medicine, Chiba University, \\ Chiba 260-8670, Japan; ${ }^{9}$ Department of Hematology/ \\ Oncology, Gunma Children's Medical Center, Gunma \\ 377-8577, Japan; ${ }^{10}$ Department of Physiology, School of \\ Medicine, Keio University, Tokyo 160-8582, Japan; \\ ${ }^{11}$ Department of Developmental Biology, Graduate School \\ of Medicine, Tohoku University, Sendai 980-8575, Japan; \\ ${ }^{12}$ Department of Cell Therapy and Transplantation Medicine, \\ University of Tokyo Hospital, Tokyo 113-8655, Japan
}

Common molecular machineries between hematopoietic stem cell (HSC) maintenance and leukemia prevention have been highlighted. The tumor suppressor Fbxw7 (F-box and WD-40 domain protein 7), a subunit of an SCF-type ubiquitin ligase complex, induces the degradation of positive regulators of the cell cycle. We demonstrate that inactivation of $F b x w 7$ in hematopoietic cells causes premature depletion of HSCs due to active cell cycling and p53-dependent apoptosis. Interestingly, Fbxw 7 deletion also confers a selective advantage to cells with suppressed p53 function, eventually leading to development of T-cell acute lymphoblastic leukemia (T-

[Keywords: Fbxw7; c-Myc; Notch1; p53; hematopoiesis; T-ALL] Corresponding authors.

${ }^{13}$ E-MAIL oike@gpo.kumamoto-u.ac.jp; FAX 81-96-373-5145.

${ }^{14}$ E-MAIL sudato@sc.itc.keio.ac.jp; FAX 81-3-5363-3474.

Article published online ahead of print. Article and publication date are online at http://www.genesdev.org/cgi/doi/10.1101/gad.1621808.

\begin{abstract}
ALL). Thus, Fbxw7 functions as a fail-safe mechanism against both premature HSC loss and leukemogenesis.

Supplemental material is available at http://www.genesdev.org.

Received October 1, 2007; revised version accepted February $22,2008$.
\end{abstract}

Stem cells in various organ tissues are governed by general genetic programs that maintain their common features, including self-renewal and multipotency. Recent studies have demonstrated that it is crucial for hematopoietic stem cells (HSCs) to be quiescent for protection against oxidative stress and to sustain self-renewal capacity (Ito et al. 2004; Tothova et al. 2007). Most HSCs remain quiescent when located in the stem cell niche (Calvi et al. 2003; Zhang et al. 2003; Arai et al. 2004). Once HSCs are released from the niche, they enter the cell cycle and start to proliferate. Cell cycle kinetics of HSCs are strictly controlled by various systems to sustain blood cell production throughout life /Cheng et al. 2000). These findings indicate that precise regulation of the cell cycle in stem cells is essential to maintain stem cell phenotype. The ubiquitin-proteasome system plays a critical role in controlling physiologic events-such as cell cycle progression, apoptosis, signal transmission, and repair of DNA damage-through protein degradation (Fuchs 2005; Minella and Clurman 2005; Welchman et al. 2005; Nakayama and Nakayama 2006). Fbxw7 (F-box and WD-40 domain protein 7)-also known as Fbw7, Sel10, hCdc4, or hAgo-is an SCF ubiquitin ligase component catalyzing ubiquitination of Myc, cyclin E, Notch, and c-Jun, all of which positively regulate the cell cycle. We hypothesized that a protein like Fbxw7 may play a pivotal role in controlling the HSC cell cycle and maintaining normal hematopoiesis. Tetzlaff et al. (2004) and we (Tsunematsu et al. 2004) independently reported that Fbxw7-deficient mice die at embryonic day 10.5 and exhibit deficiencies in hematopoietic and vascular development, suggesting that Fbxw7 functions in hematopoiesis. Mutations in FBXW7 have been detected in certain human malignancies, including T-cell acute lymphoblastic leukemia (T-ALL) (Spruck et al. 2002; EkholmReed et al. 2004; Mao et al. 2004; Maser et al. 2007). Recent reports have highlighted the existence of molecules operating differentially in the self-renewal of both normal tissue stem cells and cancer stem cells (Yilmaz et al. 2006; Zhang et al. 2006). In this study, we addressed this issue by examining the effect of $\mathrm{Fbxw}_{7} 7$ deletion on the maintenance of HSCs and leukemogenesis. Here, we demonstrate that inactivation of Fbxw7 in bone marrow (BM) HSCs leads to premature depletion of normal HSCs due to active cell cycling and promotes T-ALL due to a compromised p53 response. Thus, Fbxw7 acts as a critical fail-safe against premature loss of HSCs and development of T-ALL (Supplemental Fig. S1).

\section{Results and Discussion}

We examined Fbxw7 expression by quantitative RTPCR in various hematopoietic lineages sorted from adult mouse tissues. Fbxw7 was expressed abundantly in most of the hematopoietic cells tested, including $\mathrm{Lin}^{-} \mathrm{Sca}-1^{+} \mathrm{c}-$ $\mathrm{Kit}^{+}$(LSK) CD34- HSCs (Supplemental Fig. S2). 

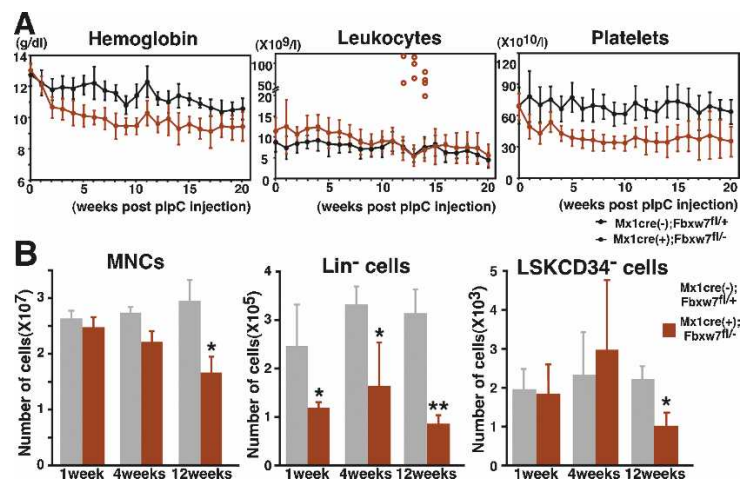

Figure 1. Fbxw7 is essential to maintain the adult hematopoietic pool. (A) Peripheral blood cell counts of Fbxw7-deficient (red closed circles, $n=15$ ) and control (black closed circles, $n=15$ ) mice after pIpC treatment. Results are shown as means \pm SD. Red open circles indicate white blood cell counts of mice developing leukemia. $(B)$ Absolute numbers of MNCs, Lin ${ }^{-}$cells, and LSK CD34- HSCs in Fbxw7-deficient and control BM at 4, 8, and 12 wk after pIpC treatment. Results are shown as means \pm SD from six to eight independent experiments. $\left.\left(^{\star}\right) P<0.05 ;{ }^{\star \star}\right) P<0.01$.

To investigate the role of Fbxw7 in adult tissues, we generated $F b x w 7^{f l / f 1}$ mice in which $F b x w 7$ was deleted conditionally in targeted cells (Onoyama et al. 2007). In this study, we created $M x-1-C r e(+) ; F b x w 7^{f l /}$ mice by crossing $M x-1-C r e(+) ; F b x w 7^{+/-}$mice and $F b x w 7^{f l / f l}$ mice in order to investigate the role of Fbxw7 in hematopoietic cells. pIpC was injected into the 8 -wk-old $M x-1$ Cre(+);Fbxw $7^{f 1-}$ mice every other day for $1 \mathrm{wk}$ to induce Cre expression and thereby delete Fbxw7 in their hematopoietic cells. $M x-1-C r e(-) ; F b x w 7^{f l /+}$ littermates treated with pIpC served as controls. Mx-1-Cre(-);Fbxw $7^{f l-}$ and $M x-1-C r e(+) ; F b x w 7^{f l /+}$ littermates showed no significant difference from control mice in our analysis (data not shown). We confirmed that Fbxw7 deletion was induced in BM cells as early as $3 \mathrm{~d}$ after pIpC treatment (Supplemental Fig. S3A).

We analyzed peripheral blood cell counts of Fbxw7deficient mice. Levels of hemoglobin and platelets were markedly lower compared with controls immediately after pIpC treatment (Fig. 1A). However, these levels stopped decreasing $\sim 12-16$ wk after pIpC treatment. Genomic PCR analysis detected a significant amount of unexcised floxed allele in Fbxw7-deficient hematopoietic cells 16 wk after pIpC treatment (Supplemental Fig. S3B). These data suggest that hematopoiesis was compromised in the absence of Fbxw7 after pIpC treatment, whereas the few cells that retained the unexcised Fbxw 7 allele gradually competed out the Fbxw7-deficient hematopoietic cells.

The number of the Lin $^{-}$cells was also reduced immediately in Fbxw7-deficient BM after pIpC treatment. In contrast, the number of BM mononuclear cells (MNCs) and LSK CD34- HSCs remained within normal range at the beginning of treatment but was decreased significantly by 12 wk of pIpC treatment (Fig. 1B). The size of the thymus was also reduced significantly in Fbxw7-deficient mice (data not shown). To determine whether Fbxw7 is essential for HSC differentiation, we examined the proportion of differentiated cells in Fbxw7-deficient BM by flow cytometry. Although a portion of lymphoid cells was decreased slightly, populations of cells sufficient to generate both mature myeloid and lymphoid cells were observed in Fbxw7-deficient BM 4 wk after pIpC treatment (Supplemental Fig. S4A). Moreover, in vitro assays revealed that colony-forming capacity was comparable between control and Fbxw7-deficient LSK cells (Supplemental Fig. S4B). Morphological analysis of colonies demonstrated the full range of differentiation capacity in Fbxw7-deficient LSK cells along the myeloid lineage (data not shown). These data indicate that Fbxw7 is dispensable for multilineage terminal differentiation.

To examine the repopulating capability of Fbxw7-deficient HSCs, we transplanted 1500 LSK BM cells from Fbxw7-deficient mice or littermate controls 4 wk after pIpC treatment into lethally irradiated recipients using $4 \times 10^{5}$ normal BM mononuclear competitor cells. Although there was no significant difference in the proportion of LSK CD34- HSCs within injected LSK cells, Fbxw7-deficient LSK cells showed severely impaired repopulating capacity (Fig. 2A, left). To confirm whether Fbxw7 intrinsically regulates HSC repopulating capability, we transplanted $4 \times 10^{5}$ BM MNCs from either $M x^{-}$ 1-Cre(+);Fbxw $7^{f l /-}$ mice before pIpC treatment or littermate controls into lethally irradiated recipients with the same numbers of competitor cells. Two months later, we confirmed that donor cells were reconstituted in recipient $\mathrm{BM}$ and then injected the recipient mice with pIpC. Within 1 mo following pIpC treatment, Fbxw7-deficient HSCs lost long-term repopulating capability and were eventually competed out by wild-type HSCs (Fig. 2A, right).

It has been reported that most HSCs remain quiescent and that excessive acceleration of the HSC cell cycle
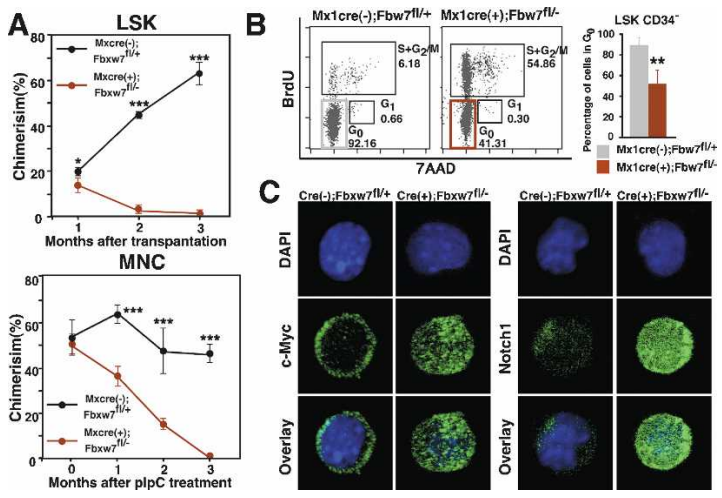

Figure 2. Reconstitution capacity and quiescence are impaired in Fbxw7-deficient HSCs. (A) Competitive repopulation analysis. (Left) Recipient Ly5.1 mice $(n=6)$ were transplanted with 1500 Fbxw7deficient or control LSK cells together with $4 \times 10^{5}$ Ly5.1 $\times$ Ly5.2 competitor BM MNCs. Donor-derived chimerism of peripheral white blood cells was analyzed monthly after transplantation. (Right) Recipient Ly5.1 mice $(n=10)$ were transplanted with $4 \times 10^{5}$ BM MNCs from Mx-1-Cre $(+) ; F b x w^{71 /-}$ mice before pIpC treatment or with controls together with the same number of Ly5.1 $\times$ Ly5. 2 competitor BM MNCs. pIpC treatment of recipient mice was performed 2 mo after transplantation. Donor-derived chimerism of peripheral white blood cells was then analyzed monthly after pIpC treatment. Results are shown as means \pm SD. $(B)$ Cell cycle status of LSK CD34- cells of Fbxw7-deficient or control mice. BrdU was administered for $3 \mathrm{~d}$ to mark cells that entered $\mathrm{S}$ phase, and 7-aminoactinomycin $\mathrm{D}$ (7-AAD) was administered to detect DNA content. Data shown are representative FACS patterns derived from three independent experiments (left) and graphs showing the mean percentage of cells in $\mathrm{G}_{0}$ (right). $(C)$ LSK cells isolated from Fbxw7deficient or control mice were stained with DAPI (blue) and anti-cMyc antibody or anti-Notch1 antibody (green). 
leads to their exhaustion (Hock et al. 2004; Ito et al. 2004). Since Fbxw7 functions as a master regulator of the cell cycle by regulating protein levels of cell cycle-related molecules, we hypothesized that a dysregulated HSC cell cycle leads to a decrease in HSC number in Fbxw7-deficient mice. To address this possibility, we examined the cell cycle status of Fbxw7-deficient HSCs using a BrdU assay. The population of Fbxw7-deficient LSK CD34- HSCs in $\mathrm{G}_{0}$ was decreased significantly compared with controls (Fig. 2B). We also monitored proliferation of single LSK CD $34^{-}$HSCs in culture for $7 \mathrm{~d}$. The frequency of cell division seen in Fbxw7-deficient HSCs was markedly increased in culture (data not shown). These data suggest that Fbxw7 functions to maintain HSC quiescence. It has been reported that c-Myc, an Fbxw7 substrate, promotes re-entry of quiescent HSCs into the cell cycle by inducing release of HSCs from the stem cell niche, leading to loss of self-renewal activity at the expense of differentiation (Wilson et al. 2004). c-Myc protein significantly accumulated in Fbxw7-deficient LSK cells (Fig. 2C), suggesting that c-Myc-induced active cell cycling of Fbxw7-deficient HSCs largely accounts for premature loss of HSCs. This hypothesis would be strongly supported by the observation that inactivation of c-Myc rescued the phenotype of hyperproliferation of DP T cells and the occurrence of lymphoma in Fbxw7deficient thymocytes (Onoyama et al. 2007). In addition, Notch1 protein, which also accumulated in Fbxw7-deficient LSK cells, might accelerate HSC differentiation (Fig. 2C; Pui et al. 1999). The levels of c-Jun, another Fbxw7 target, in Fbxw7-deficient LSK cells were equivalent to those in the control cells, suggesting that c-Jun did not contribute to the hematopoietic abnormalities in Fbxw7-deficient mice (Supplemental Fig. S5). To clarify the time course of activation of these target genes, we performed immunocytochemical staining for c-Myc and Notch at 0,72 , and $96 \mathrm{~h}$ after a single injection of pIpC $(700 \mu \mathrm{g})$ (Supplemental Fig. S6). Nuclear accumulation of c-Myc began within $72 \mathrm{~h}$, and reached plateau by $96 \mathrm{~h}$ after pIpC treatment. On the other hand, marked accumulation of Notch1 in nuclei occurred within $72 \mathrm{~h}$ after pIpC treatment. These observations indicated that ubiquitin-proteasome-dependent degradation of Notch1 and c-Myc was inhibited rapidly after $F b x w 7$ deletion by an initial single pIpC treatment. Interestingly, the accumulation of Notch1 reached plateau slightly earlier than that of c-Myc. It has been found recently that Notch1 directly regulates c-Myc transcription in normal and leukemic T cells (Weng et al. 2006). These studies suggest that the up-regulation of c-Myc expression in Fbxw7deficient HSCs might be enhanced subsequently by Notchl at the transcriptional level to some extent, in addition to increased protein stability in the absence of Fbxw7.

About $30 \%$ of Fbxw7-BM-deficient mice showed extremely severe pancytopenia $12 \mathrm{wk}$ after pIpC treatment (Fig. 3A). These mice showed a marked decrease in the number of BM MNCs, including all lineages of hematopoietic cells. It has been suggested that enhanced c-Myc expression likely activates a p53-dependent checkpoint and induces apoptosis, thereby protecting cells from hyperproliferative oncogenic signals (Zindy et al. 1998). Actually, p53 protein was markedly accumulated in $\sim 80 \%$ of Fbxw7-deficient LSK cells (Supplemental Fig. S7), while there was no difference in p53 mRNA levels between Fbxw7-deficient HSCs and the controls (Supple-

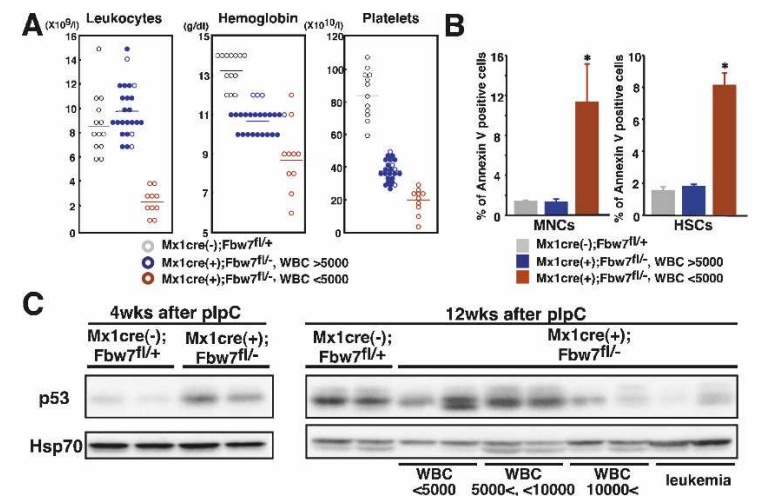

Figure 3. The fate of Fbxw7-deficient hematopoietic cells is determined by p53 expression. (A) Peripheral blood cell counts of Fbxw7deficient $(\mathrm{WBC}<5000$, red circle, $n=24$; WBC $>5000$, blue circle, $n=10$ ) and control (gray circle, $n=13$ ) mice $12 \mathrm{wk}$ after pIpC treatment. Horizontal lines indicate mean values. Closed circles indicate the data for mice that developed leukemia ultimately. $(B)$ Percentage of annexin V-positive apoptotic cells in Fbxw7-deficient (WBC $<5000$, red bar; WBC $>5000$, blue bar) and control (gray bar) BM MNCs (left) or LSK CD34- HSCs (right) 12 wk after pIpC treatment. Results are shown as means \pm SD from four independent experiments. $\left.{ }^{\star}\right) P<0.05$. (C) Western blot analysis of p53 in BM MNCs from control and Fbxw7-deficient mice $4 \mathrm{wk}$ (left) and $12 \mathrm{wk}$ (right) after pIpC treatment. Hsp70 was used as a loading control.

mental Fig. S8). Instead, Mdm2 expression was markedly down-regulated in Fbxw7-deficient HSCs at both the mRNA and protein levels (Supplemental Figs. S7, S8). Since it is believed that $\mathrm{Mdm} 2$ suppresses p53 function by promoting protein degradation via its E3 ligase activity, p53 may be up-regulated in Fbxw7-deficient HSCs, at least partly, through $\mathrm{Mdm} 2$ inactivation, although the mechanism underlying reduced $\mathrm{Mdm} 2$ protein levels remains elusive. We therefore hypothesized that the drastic reduction of hematopoietic cell number in these mice was caused not only by the dysregulated cell cycle but also by p53-dependent apoptosis. Indeed, the population of apoptotic cells in BM MNCs and HSCs increased substantially in mice with leukopenia compared with those without leukopenia (Fig. 3B). We next asked whether there is a correlation between apoptosis and p53 expression levels in Fbxw7-deficient BM cells. p53 protein levels were up-regulated in BM cells derived from all Fbxw7-deficient mice 4 wk after pIpC treatment (Fig. $3 \mathrm{C}$, left). In contrast, $\mathrm{p} 53$ protein levels were decreased in BM MNCs of Fbxw7-deficient mice that showed no leukopenia at $12 \mathrm{wk}$ after $\mathrm{pIpC}$ treatment (Fig. 3C, right). These findings suggest that the variations in white blood cell count data could be attributable to the differential modes of p53-dependent checkpoint responses, which induce apoptosis. This notion was further supported by the absence of abnormal ploidy, which could lead to apoptotic cell death, in Fbxw7-deficient BM cells /data not shown). It is well recognized that senescence as well as apoptosis is a tumorigenesis barrier (Collado et al. 2005). However, gene expression of $p 16^{\text {Ink4a }}$ was not increased in Fbxw7-deficient HSCs (Supplemental Fig. S8) and senescence-associated (SA)- $\beta$-galactosidase activity was not considerably detected in Fbxw7-deficient LSK cells (data not shown), indicating that $F b x w 7$ deletion did not promote cellular senescence.

Interestingly, more than half of the Fbxw7-deficient mice developed T-ALL (20 of 34;59\%) within $16 \mathrm{wk}$ of 

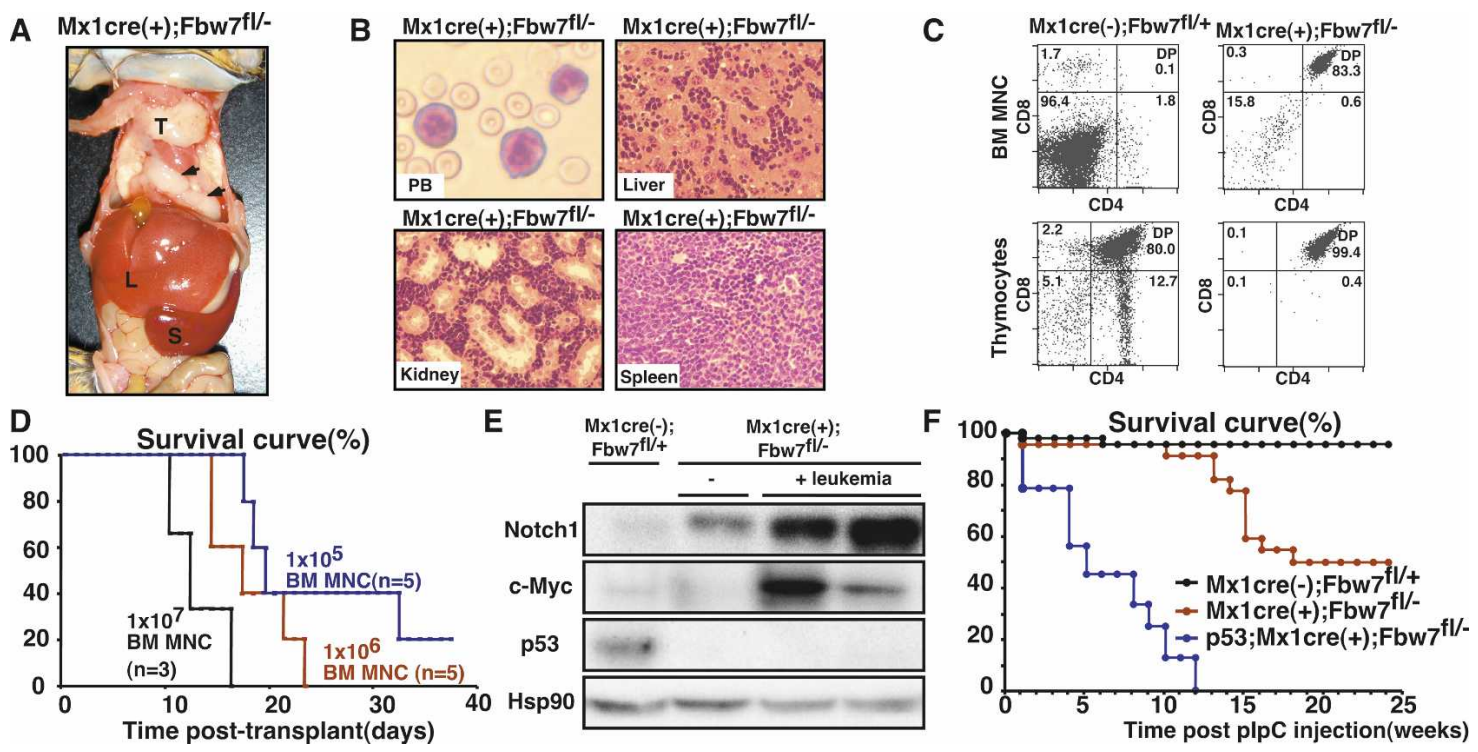

Figure 4. Fbxw7 deficiency in adult hematopoietic cells leads to T-ALL. (A) Representative autopsy of leukemic Fbxw7-deficient mice. Thymus (T), liver (L), spleen (S), and lymph nodes (arrowheads) were massively swollen. (B) Representative histology of peripheral blood (PB), liver, kidney, and spleen from leukemic Fbxw7-deficient mice. (C) FACS analysis of BM MNCs and thymocytes from leukemic Fbxw7-deficient and control mice. Data shown are representative FACS patterns derived from five independent experiments. $(D)$ Survival curves for mice injected with graded doses $\left(1 \times 10^{5}\right.$, blue; $1 \times 10^{6}$, red; or $1 \times 10^{7} \mathrm{~B}$, green) of leukemic Fbxw7-deficient BM MNCs. The average portion of $\mathrm{CD}^{+}{ }^{+} \mathrm{CD} 8{ }^{+}$leukemic blasts in leukemic Fbxw7-deficient BM MNCs was $92.4 \pm 3.1 \%$. (E) Western blot analysis of Notch, c-Myc, and p53 in thymocytes from control, Fbxw7-deficient, and leukemic Fbxw7-deficient mice. (F) Survival curves for Fbxw7-deficient $($ red, $n=23)$, $p 53^{-1}$ -;Fbxw7-deficient (blue, $n=9$ ), and control (black, $n=25$ ) mice after pIpC treatment.

pIpC treatment (Fig. 4A). In the leukemic mice, lymphoid blasts aggressively invaded BM, liver, spleen, thymus, and kidney (Fig. 4B). Flow cytometry analysis showed that blasts expressed both CD4 and CD8 (Fig. 4C). Furthermore, irradiated mice transplanted with these leukemia cells died of T-ALL within 1 mo (Fig. 4D), indicating $\mathrm{Fbxw} 7$ deletion promoted generation of T-ALL-initiating cells. Most of the Fbxw7-deficient mice that did not exhibit leukopenia at 12 wk after pIpC developed T-ALL (20 of 24; $83 \%$ ) within 16 wk of pIpC treatment, while no $\mathrm{Fbxw7}$-deficient mice that exhibited leukopenia led to leukemia (Fig. 3A).

Importantly, leukemic cells of Fbxw7-deficient mice displayed significant accumulation of Notch1 and c-Myc proteins (Fig. 4E). Interestingly, the Notch1/c-Myc signaling axis is causally linked to T-ALL development (Weng et al. 2006). These findings suggest that accumulated Notch1 and c-Myc proteins in Fbxw7-deficient BM cells caused extrathymic development of T-lineage cells and induced T-ALL. Of note was that $\mathrm{p} 53$ protein expression was repressed during leukemogenesis (Fig. 4E). Fbxw7 deficiency in hematopoietic cells caused only Tcell malignancy, presumably due to Notch1 accumulation, which induces T-lineage commitment in immature cells (Look 2004; Grabher et al. 2006). Constitutive Notch1 activation in hematopoietic stem or progenitor cells blocks B-cell differentiation and expands extrathymic DP T cells in BM, eventually leading to development of T-ALL in BM (Pui et al. 1999). In human T-ALL, Notch 1 is the most commonly mutated gene (Weng et al. 2004). While $\mathrm{Fbxw7}$-deficient mice did not show a block in B-cell differentiation (data not shown), they did exhibit extrathymic development of DP T cells and T-ALL. In this regard, T-ALL development can be attributed largely to an activated Notch pathway in Fbxw7-defi- cient mice. In contrast, deletion of $F b x w 7$ in the thymus of Lck-cre (+);Fbxw $7^{f l / f 1}$ mice (Fbxw7-thymus-deficient mice) promotes development of $\mathrm{CD} 4^{+} \mathrm{CD} 8^{+}$lymphoblastic thymic lymphoma without BM invasion (Onoyama et al. 2007), suggesting that the developmental stage at which $\mathrm{Fbxw} 7$ deletion occurs defines T-lineage tumor types; i.e., leukemia versus lymphoma.

To determine whether p53 inactivation promotes leukemogenesis in Fbxw7-deficient mice, we generated

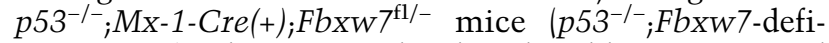
cient mice). These mice developed milder anemia and thrombocytopenia than did Fbxw7-deficient mice and exhibited considerably greater numbers of peripheral leukocytes after pIpC treatment (Supplemental Fig. S9), indicating that $\mathrm{p} 53$-dependent apoptosis contributes to the reduction of Fbxw7-deficient BM. All $p 53^{-1-}$;Fbxw7deficient mice developed T-cell malignancies with a much shorter latency and died within $12 \mathrm{wk}$, suggesting that a p53-dependent checkpoint suppresses leukemogenesis in Fbxw7-deficient mice (Fig. 4F). During our observation of $25 \mathrm{wk}$, two of $13 \mathrm{p} 53^{-\gamma-}$ mice died of T-cell thymic lymphoma, but none of them developed T-ALL (data not shown). These findings suggest that deletion of Fbxw7 provides strong selection of hematopoietic cells that harbor suppressed p53 function. Several studies demonstrate that p53 expression or function is suppressed during tumorigenesis by oncogenes including Notch and c-Myc (Eischen et al. 1999; Beverly et al. 2005). Eischen et al. (1999) have reported that c-Myc activates the p19 ${ }^{\text {Arf }}-\mathrm{Mdm} 2-\mathrm{p} 53$ tumor suppressor pathway, enhancing p53-dependent apoptosis, and strongly selects for subsequently spontaneous p53 inactivation, resulting in canceling its protective checkpoint function and the occurrence of tumorigenesis, such as lymphoma. These findings support the idea that p53 inactivation is 
an essential event in leukemogenesis in Fbxw7-deficient hematopoietic cells. Although p19 ${ }^{\text {Arf }}$, an upstream p53 regulator, was significantly elevated and $\mathrm{Mdm} 2$ was reduced, p53 was significantly inactivated in Fbxw7-deficient leukemic cells (Supplemental Fig. S10). p53 exerts negative feedback control on $\mathrm{p} 19^{\text {Arf }}$ through its interactor, CARF (Kamrul et al. 2007). Thus, elevated p19 Arf protein levels seen in T-ALL cells further support the fact that p53 function is strongly inhibited during leukemogenesis. Although the precise mechanism underlying reduced p53 protein levels remains elusive, p53 degradation via a proteasome-dependent pathway is likely compromised in the absence of Fbxw7. Loss of Fbxw7 also induces genomic instability and genomic alterations (Rajagopalan et al. 2004), which may also initiate leukemogenesis in Fbxw7-deficient mice.

These mouse phenotypes led us to analyze FBXW7 mutations in human T-ALL patients. Mutation of FBXW7 in the WD40 domain, a crucial site for protein targeting, was observed in eight of 44 cases, and most mutations (seven of eight; $88 \%$ ) were heterozygous (Supplemental Table S1), suggesting that FBXW7 loss of function is tightly associated with T-ALL development in humans and that $F B X W 7$ acts in a haplo-insufficient manner as a tumor suppressor gene in humans as in mice. On the other hand, gain-of-function mutations in NOTCH1 (N-terminal [HD-N] and C-terminal [HD-C] heterodimerization domains and PEST domains) (Weng et al. 2004; Grabher et al. 2006) were observed in 19 of 44 cases. Interestingly, most T-ALL cases with the FBXW7 mutation (six of eight; $75 \%$ ) harbored the NOTCH1 mutations as well, but which mutation is more primary in these T-ALL cases could not be determined sufficiently in the current study. $c-M y c$ is a direct Notch 1 target in Notch-dependent T-ALL (Weng et al. 2006). Since NOTCH and c-MYC are FBXW7 targets, NOTCH1-independent c-MYC up-regulation could further contribute to leukemogenesis in T-ALL with both NOTCH1 and FBXW7 mutations. Moreover, given the two samples with $F B X W 7$ mutations had wild-type NOTCH1 (two of eight; $25 \%$ ), FBXW7 mutations do not necessarily require the NOTCH1 mutations as we presented in a mouse model in this study.

This is the first report of FBXW7 mutations in Japanese T-ALL patients, representing a quite even population compared with the other races that were reported previously. It has been well established that Notch1 gain-of-function mutation is considered as a primary cause to develop T-ALL, because somatic activating mutations of Notch 1 have been identified in $>50 \%$ of all T-ALL cases and are found in all previously defined T-ALL subtypes (Grabher et al. 2006). On the other hand, our analysis in this study revealed that FBXW7 single mutation was seen in $4.5 \%$ (two of 44 ) of Japanese T-ALL patients. This frequency is slightly lower than that of T-ALL patients in North America: 6.5\% (six of 92) (Thompson et al. 2007) and 6.3\% (two of 32) (Maser et al. 2007). These findings indicate that there is a genomic type of $F B X W 7$ single mutation in T-ALL patients infrequently but universally, suggesting the existence of $\mathrm{T}$ ALL that does not bear a NOTCH1 mutation. We believe that it is noteworthy that we clearly demonstrated, through our analysis of a murine T-ALL model, the mechanism in which $F B X W 7$ loss of function could be a primary cause for developing T-ALL similar to a NOTCH1 gain-of-function mutation.
Recently, an array-based comparative genome hybridization (array-CGH) study indicated that FBXW7 and PTEN are commonly mutated in human T-ALL (Maser et al. 2007). It was reported previously that Pten deletion induces abnormal active cell cycling of HSCs, leading to their premature loss. Pten deletion also results in generation of leukemia-initiating cells. Inhibition of mTOR by rapamycin not only depletes leukemia-initiating cells but also restores normal HSC function (Yilmaz et al. 2006). Similar to Pten, Fbxw7 has distinct effects on normal stem cells and cancer stem cells within the same tissue (Supplemental Fig. S1). Loss-of-function mutations in these genes in normal HSCs are likely prerequisites for generation of cancer stem cells.

\section{Materials and methods}

$\mathrm{Mx}-1-\mathrm{Cre}(+)$; $\mathrm{Fbxw}^{+/-}$mice were obtained by mating $\mathrm{Fbxw}^{+/-}$mice (Tsunematsu et al. 2004) with interferon-inducible $M x-1$-Cre transgenic mice. To generate $M x-1-C r e(+) ; F b x w 7^{f l-}$ (Fbxw7-deficient) and $M x-1-$ Cre(-);Fbxw $7^{+/ f 1}$ (control) mice, $M x-1-C r e(+) ; F b x w 7^{+/-}$mice were crossed with $F b x w 7^{f l / f 1}$ mice (Onoyama et al. 2007). To induce Cre, mice received $500 \mu \mathrm{g}$ of pIpC intraperitoneally on three alternate days. C57BL/6-Ly5.1 congenic mice were purchased from Sankyo-Lab Service and C57BL/6Ly5.1/Ly5.2 $\mathrm{F}_{1}$ mice were used for competitive reconstitution assays. Animal care was in accordance with the guidance of Keio University for animal and recombinant DNA experiments. See the Supplemental Material for additional procedures.

\section{Acknowledgments}

We are grateful to Dr. Klaus Rajewsky (Harvard Medical School) for kindly providing us with $M x$-1-Cre transgenic mice, and to Dr. Stephen J. Elledge (Harvard Medical School) for helpful advice. We thank Drs. Kristen E. Hurov and Shuhei Matsuoka for critical assistance in manuscript preparation, and A. Ono, K. Fukushima, T. Okawa, and S. Suzuki for technical assistance with the experiments. This work was supported by grants-in-aid for Specially Promoted Research and Scientific Research (C) from the Ministry of Education, Culture, Sports, Science and Technology of Japan.

\section{References}

Arai, F., Hirao, A., Ohmura, M., Sato, H., Matsuoka, S., Takubo, K., Ito, K., Koh, G.Y., and Suda, T. 2004. Tie2/angiopoietin-1 signaling regulates hematopoietic stem cell quiescence in the bone marrow niche. Cell 118: 149-161.

Beverly, L.J., Felsher, D.W., and Capobianco, A.J. 2005. Suppression of p53 by Notch in lymphomagenesis: Implications for initiation and regression. Cancer Res. 65: 7159-7168.

Calvi, L.M., Adams, G.B., Weibrecht, K.W., Weber, J.M., Olson, D.P., Knight, M.C., Martin, R.P., Schipani, E., Divieti, P., Bringhurst, F.R., et al. 2003. Osteoblastic cells regulate the haematopoietic stem cell niche. Nature 425: 841-846.

Cheng, T., Rodrigues, N., Shen, H., Yang, Y., Dombkowski, D., Sykes, M., and Scadden, D.T. 2000. Hematopoietic stem cell quiescence maintained by p21cip1/waf1. Science 287: 1804-1808.

Collado, M., Gil, J., Efeyan, A., Guerra, C., Schuhmacher, A.J., Barradas, M., Benguria, A., Zaballos, A., Flores, J.M., Barbacid, M., et al. 2005. Tumour biology: Senescence in premalignant tumours. Nature 436: 642 .

Eischen, C.M., Weber, J.D., Roussel, M.F., Sherr, C.J., and Cleveland, J.L. 1999. Disruption of the ARF-Mdm2-p53 tumor suppressor pathway in Myc-induced lymphomagenesis. Genes \& Dev. 13: 2658-2669.

Ekholm-Reed, S., Spruck, C.H., Sangfelt, O., van Drogen, F., MuellerHolzner, E., Widschwendter, M., Zetterberg, A., and Reed, S.I. 2004. Mutation of hCDC4 leads to cell cycle deregulation of cyclin E in cancer. Cancer Res. 64: 795-800.

Fuchs, S.Y. 2005. Tumor suppressor activities of the Fbw7 E3 ubiquitin ligase receptor. Cancer Biol. Ther. 4: 506-508. 
Grabher, C., von Boehmer, H., and Look, A.T. 2006. Notch 1 activation in the molecular pathogenesis of T-cell acute lymphoblastic leukaemia. Nat. Rev. Cancer 6: 347-359.

Hock, H., Hamblen, M.J., Rooke, H.M., Schindler, J.W., Saleque, S., Fujiwara, Y., and Orkin, S.H. 2004. Gfi-1 restricts proliferation and preserves functional integrity of haematopoietic stem cells. Nature 431: 1002-1007.

Ito, K., Hirao, A., Arai, F., Matsuoka, S., Takubo, K., Hamaguchi, I., Nomiyama, K., Hosokawa, K., Sakurada, K., Nakagata, N., et al. 2004. Regulation of oxidative stress by ATM is required for selfrenewal of haematopoietic stem cells. Nature 431: 997-1002.

Kamrul, H.M., Wadhwa, R., and Kaul, S.C. 2007. CARF binds to three members (ARF, p53, and HDM2) of the p53 tumor-suppressor pathway. Ann. N. Y. Acad. Sci. 1100: 312-315.

Look, A.T. 2004. Molecular pathways in T-cell acute lympho-blastic leukemia: Ramifications for therapy. Clin. Adv. Hematol. Oncol. 2: 779-780.

Mao, J.H., Perez-Losada, J., Wu, D., Delrosario, R., Tsunematsu, R., Nakayama, K.I., Brown, K., Bryson, S., and Balmain, A. 2004. Fbxw7/ $\mathrm{Cdc} 4$ is a p53-dependent, haploinsufficient tumour suppressor gene. Nature 432: 775-779.

Maser, R.S., Choudhury, B., Campbell, P.J., Feng, B., Wong, K.K., Protopopov, A., O’Neil, J., Gutierrez, A., Ivanova, E., Perna, I., et al. 2007. Chromosomally unstable mouse tumours have genomic alterations similar to diverse human cancers. Nature 447: 966-971.

Minella, A.C. and Clurman, B.E. 2005. Mechanisms of tumor suppression by the SCF(Fbw7). Cell Cycle 4: 1356-1359.

Nakayama, K.I. and Nakayama, K. 2006. Ubiquitin ligases: Cell-cycle control and cancer. Nat. Rev. Cancer 6: 369-381.

Onoyama, I., Tsunematsu, R., Matsumoto, A., Kimura, T., de Alboran, I.M., Nakayama, K., and Nakayama, K.I. 2007. Conditional inactivation of Fbxw7 impairs cell-cycle exit during $\mathrm{T}$ cell differentiation and results in lymphomatogenesis. J. Exp. Med. 204: 2875-2888.

Pui, J.C., Allman, D., Xu, L., DeRocco, S., Karnell, F.G., Bakkour, S., Lee, J.Y., Kadesch, T., Hardy, R.R., Aster, J.C., et al. 1999. Notch1 expression in early lymphopoiesis influences $\mathrm{B}$ versus $\mathrm{T}$ lineage determination. Immunity 11: 299-308.

Rajagopalan, H., Jallepalli, P.V., Rago, C., Velculescu, V.E., Kinzler, K.W., Vogelstein, B., and Lengauer, C. 2004. Inactivation of hCDC4 can cause chromosomal instability. Nature 428: 77-81.

Spruck, C.H., Strohmaier, H., Sangfelt, O., Muller, H.M., Hubalek, M. Muller-Holzner, E., Marth, C., Widschwendter, M., and Reed, S.I. 2002. hCDC4 gene mutations in endometrial cancer. Cancer Res. 62: 4535-4539.

Tetzlaff, M.T., Yu, W., Li, M., Zhang, P., Finegold, M., Mahon, K., Harper, J.W., Schwartz, R.J., and Elledge, S.J. 2004. Defective cardiovascular development and elevated cyclin E and Notch proteins in mice lacking the Fbw7 F-box protein. Proc. Natl. Acad. Sci. 101: 3338-3345.

Thompson, B.J., Buonamici, S., Sulis, M.L., Palomero, T., Vilimas, T., Basso, G., Ferrando, A., and Aifantis, I. 2007. The SCFFBW7 ubiquitin ligase complex as a tumor suppressor in T cell leukemia. J. Exp. Med. 204: $1825-1835$.

Tothova, Z., Kollipara, R., Huntly, B.J., Lee, B.H., Castrillon, D.H., Cullen, D.E., McDowell, E.P., Lazo-Kallanian, S., Williams, I.R., Sears, C., et al. 2007. FoxOs are critical mediators of hematopoietic stem cell resistance to physiologic oxidative stress. Cell 128: 325 339.

Tsunematsu, R., Nakayama, K., Oike, Y., Nishiyama, M., Ishida, N., Hatakeyama, S., Bessho, Y., Kageyama, R., Suda, T., and Nakayama, K.I. 2004. Mouse Fbw7/Sel-10/Cdc4 is required for notch degradation during vascular development. J. Biol. Chem. 279: 9417-9423.

Welchman, R.L., Gordon, C., and Mayer, R.J. 2005. Ubiquitin and ubiquitin-like proteins as multifunctional signals. Nat. Rev. Mol. Cell Biol. 6: 599-609.

Weng, A.P., Ferrando, A.A., Lee, W., Morris, J.P.t., Silverman, L.B. Sanchez-Irizarry, C., Blacklow, S.C., Look, A.T., and Aster, J.C. 2004. Activating mutations of NOTCH1 in human T cell acute lymphoblastic leukemia. Science 306: 269-271.

Weng, A.P., Millholland, J.M., Yashiro-Ohtani, Y., Arcangeli, M.L., Lau, A., Wai, C., Del Bianco, C., Rodriguez, C.G., Sai, H., Tobias, J., et al. 2006. c-Myc is an important direct target of Notch1 in T-cell acute lymphoblastic leukemia/lymphoma. Genes \& Dev. 20: 2096-2109.
Wilson, A., Murphy, M.J., Oskarsson, T., Kaloulis, K., Bettess, M.D., Oser, G.M., Pasche, A.C., Knabenhans, C., Macdonald, H.R., and Trumpp, A. 2004. c-Myc controls the balance between hematopoietic stem cell self-renewal and differentiation. Genes \& Dev. 18: $2747-$ 2763.

Yilmaz, O.H., Valdez, R., Theisen, B.K., Guo, W., Ferguson, D.O., Wu, H., and Morrison, S.J. 2006. Pten dependence distinguishes haematopoietic stem cells from leukaemia-initiating cells. Nature 441: 475-482.

Zhang, J., Niu, C., Ye, L., Huang, H., He, X., Tong, W.G., Ross, J., Haug, J., Johnson, T., Feng, J.Q., et al. 2003. Identification of the haematopoietic stem cell niche and control of the niche size. Nature 425: $836-841$.

Zhang, J., Grindley, J.C., Yin, T., Jayasinghe, S., He, X.C., Ross, J.T. Haug, J.S., Rupp, D., Porter-Westpfahl, K.S., Wiedemann, L.M., et al 2006. PTEN maintains haematopoietic stem cells and acts in lineage choice and leukaemia prevention. Nature 441: 518-522.

Zindy, F., Eischen, C.M., Randle, D.H., Kamijo, T., Cleveland, J.L., Sherr, C.J., and Roussel, M.F. 1998. Myc signaling via the ARF tumor suppressor regulates p53-dependent apoptosis and immortalization. Genes \& Dev. 12: 2424-2433. 


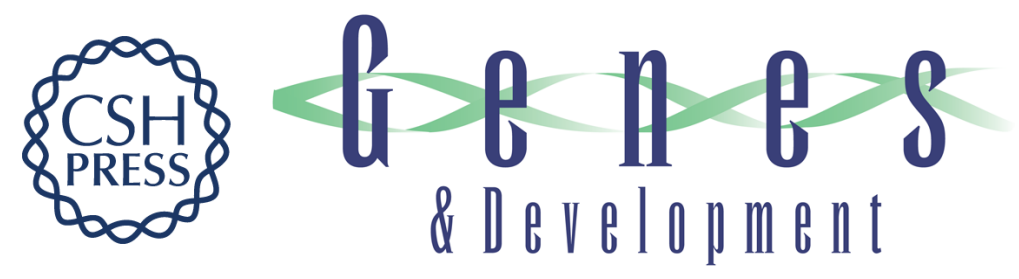

\section{Fbxw7 acts as a critical fail-safe against premature loss of hematopoietic stem cells and development of T-ALL}

Sahoko Matsuoka, Yuichi Oike, Ichiro Onoyama, et al.

Genes Dev. 2008, 22: originally published online March 26, 2008

Access the most recent version at doi:10.1101/gad.1621808

\section{Supplemental http://genesdev.cshlp.org/content/suppl/2008/03/26/gad.1621808.DC1 \\ Material}

Related Content

Self-renewal versus transformation: Fbxw7 deletion leads to stem cell activation and leukemogenesis

John M. Perry and Linheng Li

Genes Dev. May , 2008 22: 1107-1109

References This article cites 33 articles, 13 of which can be accessed free at: http://genesdev.cshlp.org/content/22/8/986.full.html\#ref-list-1

Articles cited in:

http://genesdev.cshlp.org/content/22/8/986.full.html\#related-urls

\section{License}

Email Alerting

Service

Receive free email alerts when new articles cite this article - sign up in the box at the top right corner of the article or click here.

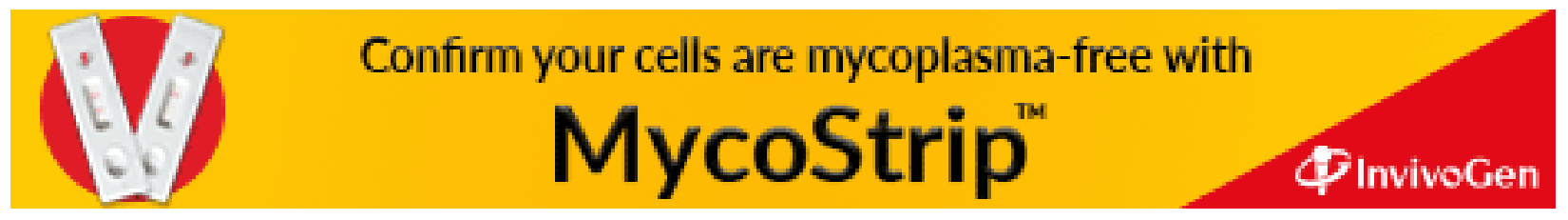

